Revista De Metalurgia 50(4)

Octubre-Diciembre 2014, e033

ISSN-L: 0034-8570

doi: http://dx.doi.org/10.3989/revmetalm.033

\title{
REVISIÓN
}

\section{La metalurgia del mercurio en Almadén: desde los hornos de aludeles a los hornos Pacific}

\author{
José Tejero-Manzanares ${ }^{\mathrm{a}, \bowtie}$, Ignacio Garrido Sáenz ${ }^{\mathrm{a}}$, Francisco Mata Cabrera ${ }^{\mathrm{a}}$, María Luisa Rubio Mesas ${ }^{\mathrm{a}}$ \\ ${ }^{a}$ Universidad de Castilla-La Mancha, Dpto. de Mecánica Aplicada e Ingeniería de Proyectos, \\ Plaza de Manuel Meca, 1. 13400, Almadén (Ciudad Real), España \\ Autor para la correspondencia: jose.tejero@uclm.es
}

Enviado: 29 Abril 2013; Aceptado: 23 Agosto 2013; Publicado on-line: 15 D iciembre 2014

\begin{abstract}
RESUMEN: Este artículo profundiza en el conocimiento de los diferentes tipos de hornos de tostación de cinabrio, utilizados en la metalurgia del mercurio a lo largo de los siglos de explotación de las Minas de Almadén (España). Algunos de ellos forman parte de nuestro patrimonio histórico industrial y han contribuido a que todo el amplio legado tecnológico de estas minas haya sido nombrado recientemente Patrimonio de la Humanidad por la UNESCO. Este trabajo pretende abordar el largo bagaje de la actividad metalúrgica desde los hornos de aludeles hasta los hornos Pacific, primera y última de las tecnologías empleadas en producción a escala industrial. Se profundiza en aquellos aspectos más relevantes que tienen que ver con la tipología, evolución y número de hornos existentes en cada una de las épocas.
\end{abstract}

PALABRAS CLAVE: Almadén; Hornos; Mercurio; Metalurgia; Tostación del cinabrio

Citation / Cómo citar este artículo: Tejero-Manzanares, J., Garrido Sáenz, I., Mata Cabrera, F., Rubio Mesas, M.L. (2014) "La metalurgia del mercurio en Almadén: desde los hornos de aludeles a los hornos Pacific". Rev. Metal. 50(4): e033. doi: http://dx.doi.org/10.3989/revmetalm.033.

\begin{abstract}
Metallurgy of mercury in Almaden: from aludel furnaces until Pacific furnaces: This paper shows the different types of furnaces for roasting cinnabar, used in the metallurgy of quicksilver over the centuries of exploitation of the Almadén Mines (Spain). Some of these techniques are part of our industrial heritage. They have contributed to name UNESCO World Heritage Site the vast technological legacy of these mines recently. This research contributes to close the long way of metallurgical activity from aludel furnaces until Pacif furnaces, first and lasted technology to produce on an industrial scale. It is delved into the most relevant aspects having to do with the type, evolution and number of furnaces existing on each of the periods.
\end{abstract}

KEYWORDS: Almaden; Cinnabar roasting process; Furnaces; Mercury; Metallurgy

Copyright: (C) 2014 CSIC. This is an open-access article distributed under the terms of the Creative Commons Attribution-Non Commercial (by-nc) Spain 3.0 License.

\section{INTRODUCCIÓN}

El mercurio $(\mathrm{Hg})$ es un metal de transición que pertenece al segundo grupo del sistema periódico de los elementos, segundo subgrupo. Su número atómico es 80 y su peso atómico es 200,61. Es de color gris claro, plateado, bastante brillante, único metal líquido a temperatura ambiente y muy volátil.

Son numerosas las aplicaciones encontradas para este metal a lo largo de la historia. Durante 
siglos, el mercurio se ha usado principalmente en el proceso de amalgamación del oro y la plata, convirtiéndolo en un producto estratégico que ha reportado beneficios incalculables para España. Además, se ha utilizado en la medicina como desinfectante intestinal $\mathrm{y}$, en forma de pomadas, para tratar la piel; en odontología, en amalgamas dentales. Una de sus grandes aplicaciones se encontró en la industria armamentística en el fulminato de mercurio (ONC-Hg-CNO), que se utiliza como explosivo de iniciación por ser muy inestable y de descomposición exotérmica poco calórica. Otros usos industriales del mercurio fueron como catalizador en reacciones orgánicas, pilas, lámparas con vapor de mercurio y para esterilizar agua. También, ha formado parte de compuestos alquílicos usados en la agricultura como fungicidas con el propósito de evitar la proliferación de limo en la pulpa de papel, como plaguicidas en cultivos de árboles frutales y en el tratamiento de granos y simientes.

Las Minas de Almadén han sido explotadas de manera ininterrumpida durante más de 2000 años, siendo el mayor productor del mundo de este metal. Se estima que han producido la tercera parte del mercurio consumido por la Humanidad (Hylander y Meili, 2003). Debido a su alta toxicidad, a principios de este siglo, el Consejo de Gobierno del Programa de Naciones Unidas para el Medio Ambiente (PNUMA) implantó su Programa del Mercurio como instrumento de control internacional sobre las actividades con mercurio, quien estableció una serie de decisiones a partir de 2001 que incluían la prevención, minimización y gestión de residuos de mercurio, así como su almacenamiento a largo plazo y eliminación (López-Delgado et al., 2012). La Unión Europea, alentada y mediada por informes científicos que demuestran el potencial dañino del mercurio para la salud, puso en marcha la Estrategia Comunitaria sobre el mercurio, cuyo objeto es disminuir su impacto y sus riesgos para el medio ambiente y la salud humana. Ello, sumado a la baja rentabilidad de la explotación, derivó en la paralización en 2001 de la actividad extractiva del mineral en Almadén y, dos años más tarde, el fin de la actividad metalúrgica.

Como consecuencia de la dilatada explotación de la mina, en Almadén se emplearon un número elevado y variado de equipos diferentes para obtener el azogue por tostación del cinabrio. Los primeros métodos de producción, muy rudimentarios, conllevaban bajas producciones y problemas de salud para los operarios (Mansilla e Iraizoz, 1996). Las condiciones de trabajo eran muy duras, con altísimas exposiciones e impregnaciones de los trabajadores que desarrollaban sus labores metalúrgicas (Tejero et al., 2011). La tecnología fue evolucionando con objeto de conseguir fundamentalmente un aumento de la producción, mayores rendimientos y una mejora en las condiciones de salubridad. Una de las enfermedades más conocidas que contraía el personal que trabajaba en los hornos como consecuencia de las exposiciones mercúricas era el hidrargirismo. El estudio, prevención y curación de esta intoxicación ha sido una constante en el día a día de médicos e ingenieros, quienes trabajaban conjuntamente (Tejero y Montes, 2011).

Desde el primer tipo de hornos documentado, conocidos como hornos de xabecas e introducidos por los árabes, hasta los últimos hornos, los de soleras múltiples Pacific-Herreshof Co. de San Francisco, gran variedad se han utilizado en las instalaciones metalúrgicas de las Minas de Almadén, conocidas como Cerco de Buitrones (Tejero, 2011). De ellos, solo dos tipologías se han conservado hasta nuestros días: el par de hornos de aludeles San Eugenio y San Julián y una batería de cuatro hornos Pacific-Herreshof. Por otro lado, no existen publicaciones que traten la variedad de tecnologías empleadas para la tostación del cinabrio en las instalaciones almadenenses desde sus inicios hasta el fin de la explotación.

Mediante este trabajo, se pretende contribuir a la recuperación, protección y difusión de este vasto legado que representan los hornos de las Minas de Almadén para la tostación de cinabrio. De esta forma, se intenta evitar que las diferentes tecnologías utilizadas para extraer el mercurio pasen del olvido o de un conocimiento sesgado, a formar parte de un patrimonio al alcance de todos los que pretendan conocerlo o estudiarlo (Tejero et al., 2012).

En el caso que nos ocupa, el poner de manifiesto las distintas tecnologías metalúrgicas utilizadas a lo largo de siglos para el beneficio del mercurio en las Minas de Almadén, permite conocer de una manera más exacta y precisa tanto el funcionamiento como las razones de uso de las distintas tipologías de hornos. El reciente nombramiento de Patrimonio de la Humanidad otorgado a las Minas de Almadén por parte de la UNESCO, es una razón más para dar a conocer su patrimonio tecnológico. Se pretende conseguir dicho objetivo a través de esta investigación, resultado de una profunda revisión documental. Para su desarrollo han sido clave los legajos consultados tanto en el Fondo Antiguo del Archivo Histórico de la Fundación Almadén "Francisco Javier de Villegas" (AHFA) como en el Fondo Contemporáneo del Archivo Histórico Nacional (AHN).

La obtención tradicional del mercurio $(\mathrm{Hg})$ tiene su base en la explotación minera de su mineral, el cinabrio $(\mathrm{HgS})$, y su posterior tratamiento metalúrgico. En primer lugar, el mineral se tritura a distintas granulometrías en función del tipo de horno donde se vaya a tratar. A continuación, se procede a su tostación entre $250-300{ }^{\circ} \mathrm{C}$, reacción por la que el sulfuro de mercurio se descompone gracias a la acción reactiva del aire atmosférico, liberando 
dióxido de azufre $\left(\mathrm{SO}_{2}\right)$ más vapor mercurial que se condensa, enfría y depura en distinto grado para su comercialización (Ec. 1):

$$
\mathrm{HgS}+\mathrm{O}_{2} \rightarrow \mathrm{Hg}+\mathrm{SO}_{2}
$$

En general, la instalación metalúrgica consiste en uno o varios vasos (el horno propiamente dicho) para la tostación del cinabrio, el sistema de condensación para la obtención del azogue y el de expulsión de los gases resultantes del proceso (Tejero, 2011).

\section{ANTECEDENTES}

Zarraluqui (1934) narra que está fuera de duda que los romanos obtuvieran cinabrio de la mina de Sisapo (región de Almadén) con el fin de convertirlo en bermellón (cinabrio reducido a polvo que toma color rojo vivo) para emplearlo como pintura y que conocían los medios para extraer el mercurio del mineral para fines medicinales, amalgamar el oro y otros menesteres.

En los libros de la antigüedad, se describen métodos para la obtención de azogue a partir del cinabrio conocidos con nombres que puede que no se correspondan con sus inventores. Se trata de los métodos de Teofrasto, Dioscórides y Plinio (Escosura, 1878).

Durante la dominación árabe, se introdujo un nuevo método en la metalurgia para la obtención del mercurio (de interés en la alquimia) que siguió empleándose en la España reconquistada hasta el siglo XVII. El dispositivo conocido como hornos de xabecas (Fig. 1a), se componía de cuatro paredes verticales de planta rectangular, cerradas por una bóveda de medio punto. En la bóveda existían diversos agujeros en número variable de 18 a 24, según los casos, cuyo aspecto daba la forma de una red de donde tomó su nombre (Escosura, 1878).
El proceso consistía en preparar las ollas o xabecas (Fig. 1b) llenándolas de mineral, que previamente era triturado. A continuación, se tapaban mediante coberteras o tapaderas colocándolas, finalmente, en los agujeros de la cubierta del horno. En el interior del mismo, se colocaba la leña que ardía hasta calentar las ollas y el cinabrio introducido en ellas para que el mercurio consiguiera volatilizarse. El azogue iba depositándose en la parte superior de las ollas. Una vez enfriado el horno, se destapaban las coberteras y se obtenía el mercurio líquido envuelto en ceniza y piedras. Finalmente, la mezcla se lavaba para separar el mercurio. Hoy día no quedan apenas vestigios de los hornos árabes, sólo las numerosas ollas de xabeca que se pueden ver en los museos de Almadén.

Las ricas minas de plata de Perú (Potosí, 1545) y Nueva España (Zacatecas, 1546) fueron descubiertas, aproximadamente, a los cincuenta años de la llegada de los españoles a América y fueron puestas en explotación a los pocos años desde su hallazgo. Este hecho produjo una dependencia del mercurio en los diferentes métodos de beneficio de la plata y el oro gracias al procedimiento de amalgamación por el método de patio de Bartolomé Medina en 1555 (Mansilla, 2008).

Los hornos de xabecas fueron reemplazados poco a poco por los hornos de reverbero al no ser su rendimiento apropiado a la demanda creciente de mercurio. Construidos de ladrillo y barro, de planta rectangular y cubiertos por una bóveda semiesférica de ladrillo refractario y con chimenea, solo se emplearon para la cochura (tostación de una carga de mineral) en ollas (Fig. 1c) sin llegar a realizar la tostación del mineral en ellos directamente.

Las ollas se colocaban llenas de mineral y destapadas dentro del horno y su número variaba en función del tamaño de éste. A continuación, el horno (a)

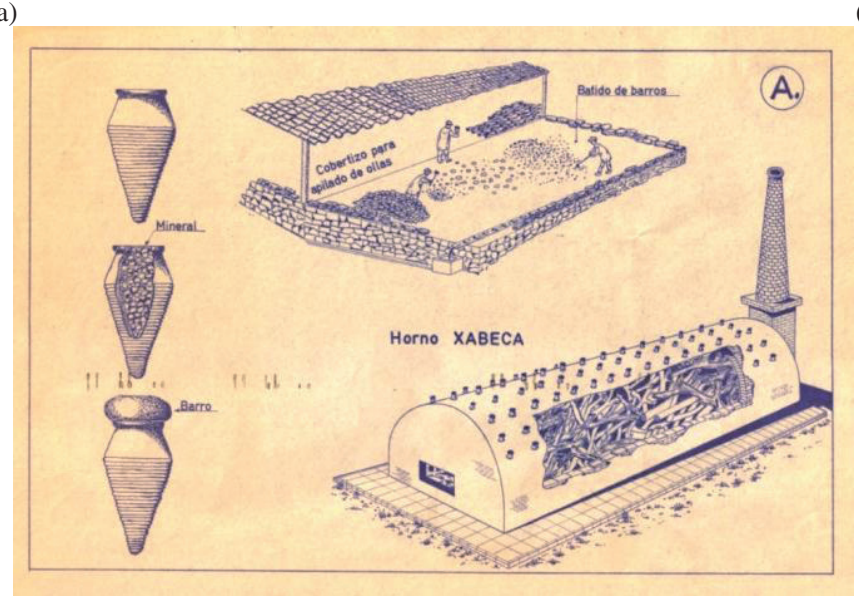

(b)

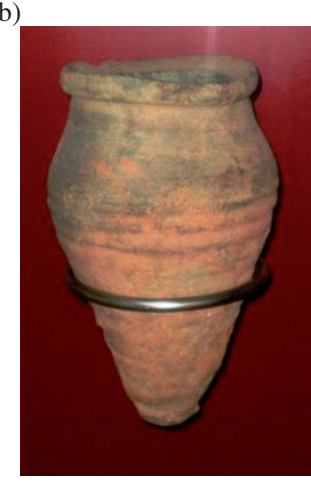

(c)

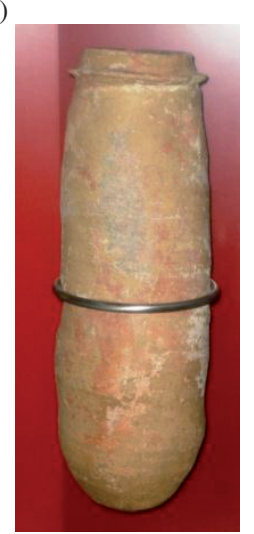

FigurA 1. a) Plano detalle hornos de xabecas (Fuente: AHFA); b) olla de xabeca; c) olla de reverbero (Parque Minero de Minas de Almadén). 
se cerraba herméticamente. El hogar o caldera se encontraba debajo de una red que sustentaba las ollas. Una vez realizada la cocción y enfriado el horno, se procedía a la obtención del mercurio. La solera tenía una ligera pendiente hacia el centro y, era ahí, donde se depositaba el mercurio al igual que en el fondo de las ollas. Para recoger el azogue condensado tanto en las ollas como en el propio horno, los operarios tenían que introducirse en su interior respirando inevitablemente los vapores mercuriales, por lo que terminaban azogándose y les provocaba, entre otros síntomas, un temblor continuado. En estos dos tipos de hornos el mineral no entraba en contacto con el combustible.

Los verdaderos sistemas de producción industrial de mercurio se desarrollaron a partir de 1646 con la introducción de los hornos de aludeles. Por ello, este trabajo se centra en los hornos metalúrgicos posteriores a los de xabecas y reverbero y, especialmente, en los hornos utilizados en la cuenca almadenense, si bien se hace referencia a los utilizados en otras cuencas mineras de Asturias (España), Huancavelica (Perú), Idria (Eslovenia) e Italia en algunas ocasiones.

\section{MATERIALES Y MÉTODOS}

La metodología empleada para la realización de este trabajo se fundamenta en las etapas de documentación, toma de datos y trabajo de campo.

En la etapa de documentación se ha realizado una revisión histórica sobre las minas y la ciudad de Almadén con el fin de obtener más vías de información. Asimismo, se ha investigado el origen, historia y evolución de los hornos en la metalurgia del mercurio. Posteriormente, se ha centrado la búsqueda en los hornos utilizados en Minas de Almadén.

Para todo ello, ha sido fundamental un examen exhaustivo tanto de la base de datos del AHFA (con sede en el Real Hospital de Mineros de San Rafael) como de la del AHN.

Seguidamente, en la etapa de toma de datos, se ha seleccionado la documentación relacionada con la evolución tecnológica de cada tipo de horno en Almadén y se han clasificado los tipos de hornos de Minas de Almadén mediante la consulta de legajos, planos y fotografías.

Durante el trabajo de campo, se ha recogido información en el Museo de la Metalurgia del Mercurio y se han analizado ejemplares de hornos y restos arqueológicos en el Cerco de Buitrones, ambos ubicados en el Parque Minero de las Minas de Almadén. También, se han visitado algunas zonas de los alrededores de la ciudad de Almadén y de la comarca donde aún quedan restos de xabecas. Por otra parte, en los AHFA se ha encontrado una enorme colección de planos pertenecientes a distintos hornos y al estado y evolución del Cerco de Destilación de las Minas de Almadén. Algunos de estos planos son de gran valor tanto por su calidad como por su antigüedad y buen estado de conservación. Además, existen allí fotografías de elevado valor por su antigüedad y memorias del Cerco de Destilación en forma de legajos. En el AHN se encontró una memoria y planos de unos hornos Almadén (de tecnología Spirek) (Tejero et al., 2012), así como los ensayos de Mr. Pellet con su invento fallido del artilugio que lleva su mismo nombre.

La interpretación conjunta de esta información documental permite identificar y ubicar espacial y temporalmente los diferentes tipos de hornos, así como descifrar con detalle el número y tipos de vasos empleados en cada uno de ellos. Además, facilita el establecimiento de una comparativa en el tiempo con los hornos de otras cuencas mineras en Asturias, Huancavelica, Idria e Italia.

La correcta utilización de esta metodología de trabajo permite sacar a la luz errores de datación como el de la fotografía fechada en 1924 tanto en el AHN como en el AHFA (Fig. 2). Este error ha dado lugar a que en distintas publicaciones se refleje que los hornos ahí representados son del tipo CermakSpirek y Spirek, cuando lo que se puede observar en primera instancia son unos hornos de Bustamante $\mathrm{y}$, en la parte superior, del centro hacia la derecha, los vetustos hornos de cámaras o de Idria y los de canales.

Puesto que los restos de los hornos de canales $o$ de Livermore, de fachada lateral parecida a los hornos Cermak-Spirek, se conservaron en el Cerco hasta 1918 (aparecen tanto en el Inventario como en un plano del Cerco de Buitrones de este mismo año), cuando fueron demolidos para construir una nueva batería de hornos Spirek, la fotografía nunca podría haberse realizado en 1924. Además, tanto en planos como en fotografías posteriores, se puede comprobar que la orientación longitudinal del edificio que acogió los hornos de tecnología Spirek es siempre de Este a Oeste y no como en este caso, que es Norte-Sur.

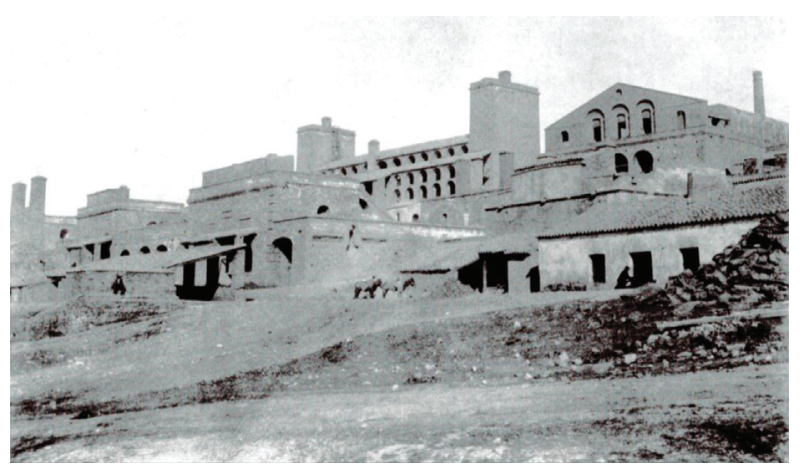

FIGURA 2. De izquierda a derecha, vista de unos hornos de aludeles, los hornos de Idria y de Livermore en el Cerco de Buitrones (Fuente: AHFA). 


\section{TIPOLOGÍA, EVOLUCIÓN Y NÚMERO DE HORNOS DESDE LOS HORNOS DE ALUDELES A LOS HORNOS PACIFIC}

\subsection{Tipologías de hornos en los siglos XVII y XVIII}

\subsubsection{Hornos de aludeles}

Con el fin de resolver los problemas que la metalurgia suscitaba en el Nuevo Mundo y en España, llegaron de América hasta Almadén los hornos de aludeles. Inventados en 1633 por el médico de Huancavelica y natural de Siruela (España) Lope de Saavedra y Barba, constituyen una de las mayores contribuciones al progreso de la metalurgia: la obtención del azogue por destilación y a escala industrial.

Sus objetivos consistían en una reducción del tiempo de cochura, ahorro de mano de obra, economía de combustible y tratamiento de minerales pobres no beneficiales mediante las ollas convencionales. El procedimiento de Saavedra sigue vigente hoy día, de modo que los métodos propuestos posteriormente para obtener el mercurio se basan, fundamentalmente, en él. Estos hornos se denominaron también busconiles en honor a la afición de su inventor de buscar minas.

El horno de aludeles fue introducido en 1646 en Almadén por Juan Alonso Bustamante, quien perfeccionó la idea de Lope de Saavedra. El primer horno construido fue el de Nuestra Señora de la Concepción, llegando a sumar un total de nueve hornos en apenas dos años. El nuevo sistema vino a revolucionar todo el proceso metalúrgico anterior, trayendo consigo grandes mejoras y, sobre todo, un aumento de la producción con la que poder abastecer el mercado de la amalgamación americana. El impulso dado a la minería del mercurio por los hornos de Bustamante provocó que fueran a Almadén los más prestigiosos científicos de la época con el fin de estudiar los nuevos hornos y efectuar mejoras para aumentar su rendimiento. Incluso, se enviaron a América dos modelos desmontados de los hornos usados en Almadén en la flota de 1677, con instrucciones muy detalladas para su construcción y operación. Es por este camino como retornaron a América, mejorados, los hornos inicialmente diseñados allí cuarenta y cuatro años antes.

La tecnología de aludeles jugó un papel vital en el beneficio del azogue (Mansilla, 2008) no solo en América, sino también en Europa, ya que además de a las Minas de Almadén y Almadenejos y otras de España como las de Alicante, Asturias, Badajoz, Castellón, Granada, etc., también se exportó a algunas regiones de Italia y, aunque con algunas modificaciones, a la ciudad de eslovena de Idria en 1750 (Betancourt, 1783).

En estos hornos, que siempre se encuentran pareados, el vaso de calcinación consiste en una cavidad cilíndrica de unos $7 \mathrm{~m}$ de largo por 1,5-2 m de ancho dividida en dos compartimentos por una rejilla llamada red formada por unos arcos de ladri1lo que soportan la carga, ambos inclinados unos $50^{\circ}$. El cuerpo inferior sirve de hogar y cenicero al mismo tiempo comunicado a la atmósfera mediante una chimenea por la que salen los productos de combustión (Fig. 3). Esta cavidad termina en forma de bóveda semiesférica con una apertura circular y cuatro ventanillas que conectan con los caños o aludeles distribuidos en dos series de ocho filas descendentes (plano de cabecera) y otras tantas ascendentes (plano de rabera) por cada horno, que hacen las veces de cámaras de condensación (Fig. 3).

Las líneas de aludeles del plano de rabera van a parar a unas camaretas situadas enfrente de la de los hornos y encargadas de recibir los gases (que contienen el azufre) que son conducidos al exterior a través de las chimeneas. Estas camaretas presentaban unas pequeñas puertas para permitir el acceso a su interior para su limpieza (Fig. 3).

El caño o aludel es un tubo de barro que tiene una longitud aproximada de $40 \mathrm{~cm}$ (Fig. 4a); en la parte más delgada, de unos $10 \mathrm{~cm}$ de diámetro $\mathrm{y}$, en la zona de la panza, de 20-22 cm. En esta zona, se puede apreciar el agujero que poseen (se orientaba hacia el suelo) y que no fue practicado desde el principio. Este agujero de unos $4 \mathrm{~mm}$ lo presentaban los aludeles situados en el plano de cabecera y tenía el fin de permitir la salida a un canal al metal líquido que se acumulaba en esta zona durante la condensación. De esta forma, se podían realizar muchas más cochuras (hasta diez) sin tener que levantar los aludeles. El mencionado canal, con una ligera pendiente, con el fin de permitir el deslizamiento del mercurio hasta un recipiente lateral, se encuentra situado en la línea convergente de los planos de cabecera y de rabera (Fig. 4b). El último aludel del plano de rabera se llamaba trompeta, era algo más corto y sin panza (Fig. 3).

En las memorias de Betancourt (1783), consta que los hornos de Bustamante introducidos en Idria sufrieron ciertas modificaciones en las camaretas que recibían los gases que contenían azufre, antes de salir al exterior a través de las chimeneas. Consistía en conducir estos gases mediante una trayectoria

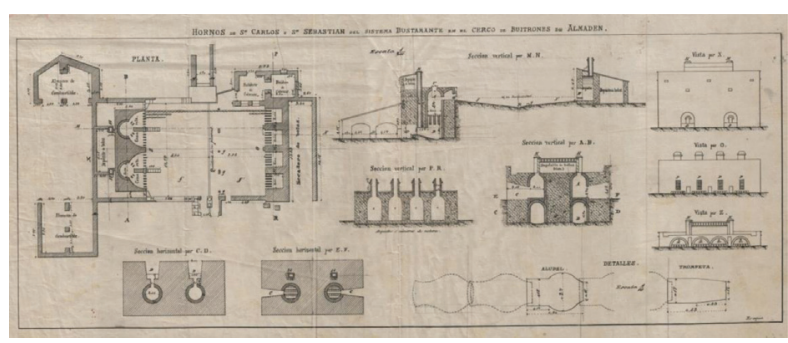

Figura 3. Plano de los hornos de aludeles o de Bustamante San Carlos y San Sebastián (Fuente: AHFA). 
(a)

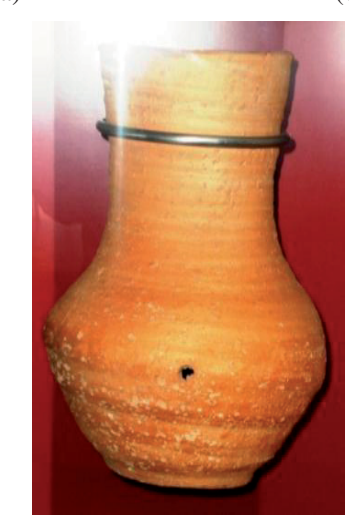

(b)

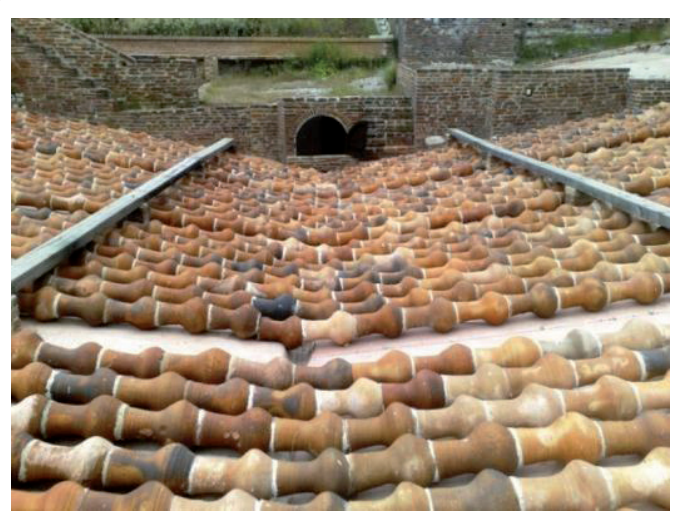

Figura 4. a) Caño o aludel de un horno de Bustamante; b) vista del canal conductor del mercurio hasta el depósito situado en su extremo.

descendente y hacerlos pasar por agua para terminar de condensar el mercurio que no lo hubiera hecho antes en su trayecto. Esta mejora se introduciría más tarde en Almadén, pero tal y como se puede comprobar en los planos que se han presentado, volvieron a su sistema inicial.

Los únicos hornos de este tipo que se han conservado en el Cerco de Buitrones son San Eugenio y San Julián. Este tipo de hornos, en funcionamiento hasta 1931, es una muestra de cómo la inventiva española de los hornos de aludeles produjeron mercurio de una manera continuada en la comarca de Almadén durante casi 300 años.

\subsection{Tipologías de hornos en el siglo XIX}

\subsubsection{Hornos de Idria}

En Idria, se modificaron los hornos de cuba de Bustamante, cambiando el sistema de aludeles por el de cámaras, dando como resultado los hornos de marcha discontinua o intermitente que llevan el nombre de esa ciudad. Fue en 1806 cuando se instalan un par de ellos en Almadén de nombres San Luis y San Carlos (Fig. 5a).

El sistema de estos hornos de cámaras, que también trabajaban pareados, consistía en un vaso central cilíndrico en el que se ubicaban el cenicero en la parte inferior, el hogar y la cámara de recepción del mineral, que debía ser previamente reducido a golpes de martillo a un tamaño adecuado para su tratamiento. Los vasos tenían una capacidad superior e igual a dos veces y media la de los de Bustamante. A diferencia de los hornos de aludeles, las arquetas del vaso se comunicaban con dos series de seis cámaras colocadas a uno y otro lado de él, en lugar de los conductos de barro. Éstas se comunicaban entre sí mediante ventanillas colocadas alternativamente abajo y arriba a fin de obligar a los gases a realizar un largo recorrido en zig-zag a modo de serpentín. Al principio, estaban fabricadas únicamente con ladrillo refractario y después se revistieron con mortero de cemento portland con el fin de facilitar el deslizamiento de las gotas de mercurio. La parte inferior tenía forma de pirámide truncada invertida donde se recogía el mercurio que era conducido mediante un pequeño canal a una pileta. La última cámara era mucho más alta que las demás porque hacía las veces de chimenea.

\subsubsection{Horno Pellet}

Las Minas de Almadén mantuvieron una intensa relación con la desconocida industria del mercurio en Asturias, lo que dio lugar a una época de influencias e intercambios de tecnología entre ambas de manera semejante a como ocurrió entre Almadén y Huancavelica. Este período es amplio, pues abarca los 80 años comprendidos entre las décadas de 1840 a 1920. En la cuenca asturiana se produjo la puesta a punto de hornos adaptados a las características del mineral de la región: aplicación consecutiva de hornos de aludeles, de cámaras, de Livermore y construcción de los allí inventados por Rodríguez y Gascue-Rodríguez (Luque y Gutiérrez, 2006).

Fue en el año 1867 cuando el ingeniero francés D. Emilio Pellet hace su primera oferta al entonces Ministro de Hacienda D. Manuel García Barzanallana, consistente en establecer un horno de su invención para beneficio de mercurio que reducía considerablemente las pérdidas. Tal cual se desprende de la $2^{\mathrm{a}}$ condición que pedía Mr. Pellet para realizar su experimento (AHFA, legajo 372), la Dirección del establecimiento almadenense propondría lo que creyera más conveniente y económico para los intereses de la Hacienda: la habilitación de uno de los hornos de Idria existentes o la construcción de uno nuevo para realizar un ensayo comparativo. Mr. Pellet encendió 
(a)

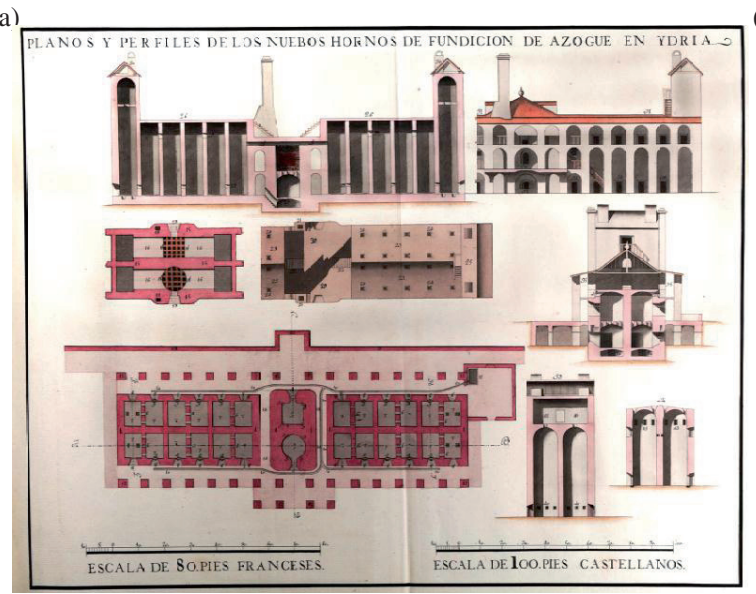

(c)

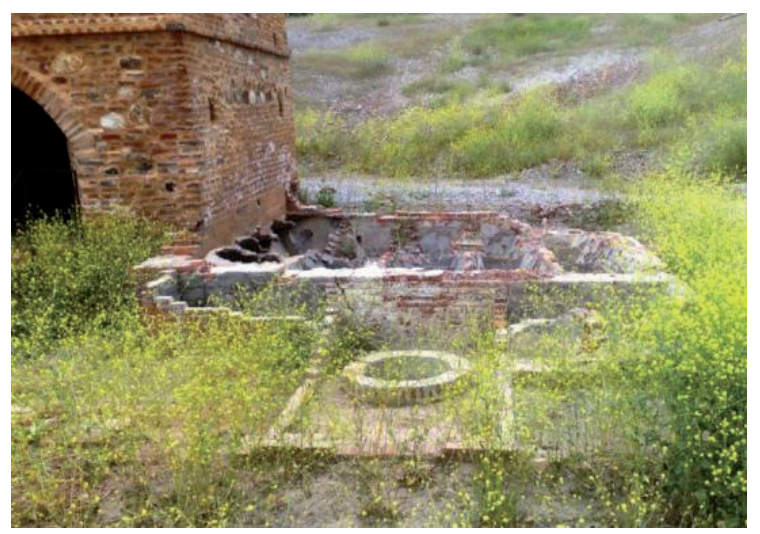

(b)

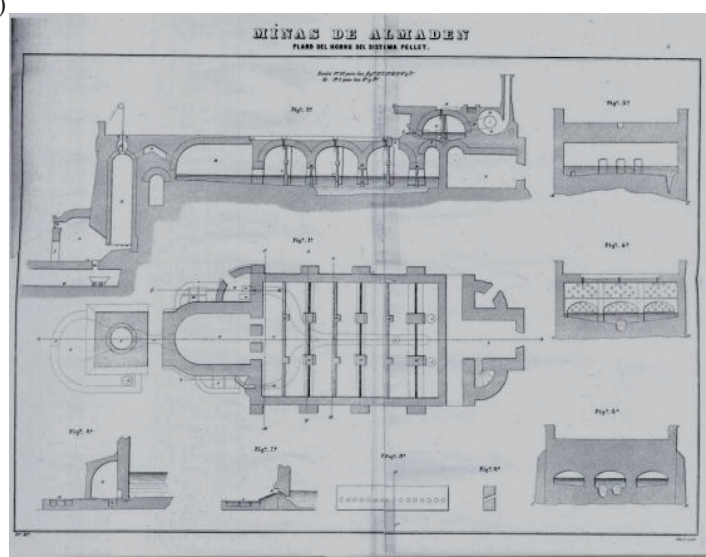

(d)

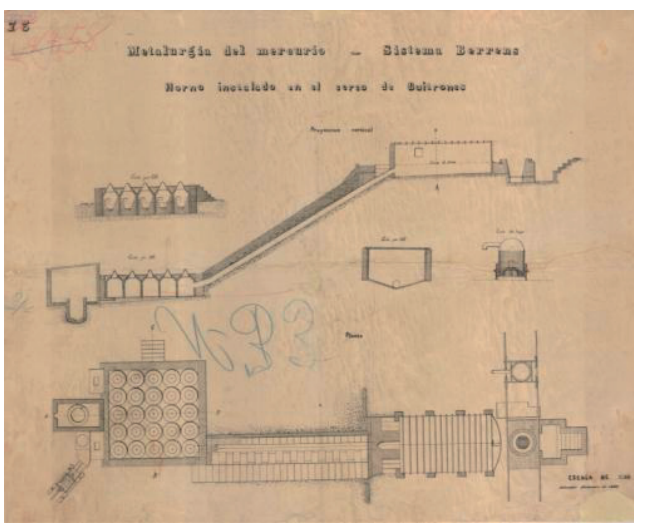

Figura 5. a) Plano de los hornos de Idria (1803). Autor: José Morete de Varela (Fuente: Museo de Ciencia y Tecnología de Madrid); b) horno Pellet (Fuente: AHFA); c) vista desde el Sur de los restos del horno Berrens junto a los hornos de tejera; d) plano del horno Berrens en el Cerco de Buitrones (1892) (Fuente: AHFA).

su horno en el Cerco de Buitrones de Almadén el 22 de marzo del año 1869. Los ensayos resultaron un auténtico fracaso y representaron un coste importante, pues duraron varios años. Se practicaron entre el horno de Pellet y los de Idria, además de los de Bustamante.

En carta dirigida al Superintendente José de Madariaga en marzo de 1870 (AHFA, Legajo 372), José de Monasterio y Correa (1870), Ingeniero e Inspector General de Minas, avanzaba el resultado de los primeros ensayos realizados antes de hacerse públicas sus memorias: el sistema Pellet suponía un coste cuatro veces y media superior al sistema de cámaras. El horno Pellet (Fig. 5b), aunque no se llegó a utilizar en las explotaciones mineras de Almadén, sí que permaneció en el Cerco de Buitrones durante bastantes años, tal y como se puede comprobar en los planos existentes de la distribución de los hornos en el Cerco de Destilación como el del año 1924. Según la Memoria del Cerco de Destilación de 1928 (AHFA, Legajo D-1525), en el mes de enero tuvo lugar la demolición de este artilugio.

\subsubsection{Horno Berrens}

Otro sistema de hornos, el de M. Berrens, se construyó por cuenta del Estado en las Minas de Almadén en 1875 en detrimento de los hornos de Idria. La huella que ha dejado este horno ha sido un conjunto de tinajillas y pocetas de decantación (Fig. 5c). Estos restos se encuentran en la parte Sureste del Centro de Buitrones, cerca del Almacén de Mercurio y junto a otros restos pertenecientes a unos hornos de tejera.

En el AHFA, fechado en diciembre de 1892, se halló un plano (Fig. 5d) que recoge cómo era este horno que, aunque no carente de errores técnicos y de omisión de algunos detalles, se observan varias cuestiones. En la proyección en planta, se puede apreciar la balsa que contiene las tinajillas (en un número de 25), coincidentes con los restos que han quedado de él. Estas tinajillas, comunicadas entre sí por la parte inferior, debían constituir el sistema de condensación de los gases mercuriales. Al lado de ellas, situada en una cota muy inferior, se encontraba la poceta, que aparece reflejada en el plano y en las fotografías, donde se recogería todo el metal. 
Los gases mercuriales eran conducidos hasta este sistema de condensación a través de un conducto en pendiente desde una cámara situada a cota superior, desde donde se le forzaría a realizar un determinado recorrido para comenzar su enfriamiento y precipitar el mercurio. El horno u hogar, de planta circular y acabado en una cúpula semiesférica, descansaba sobre unas ruedas que se desplazaban sobre unos raíles hasta acoplarse a la cámara. Estamos en condiciones de afirmar que se trata de un sistema que se aproxima mucho a los de destilación utilizados en Europa en el Medievo y reflejados en la obra de Agrícola (1556). Junto al horno Pellet, se trataba de una instalación metalúrgica con un único vaso.

\subsubsection{Hornos de canales o de Livermore}

En 1887, se instaló un nuevo horno que ya se utilizaba tanto en Asturias como en Idria y Amiata (Betancourt, 1783): el horno sistema Livermore o de canales, que es como se conocía en Almadén. De este tipo de horno de marcha continua se construyeron dos. Esta tecnología fue introducida por C.E. Livermore en la mina de Redington, California en 1874 (Schnabel, 1898; Egleston, 1890).

En el AHN, Legajo 147, se pueden comprobar los partes de producción de dos hornos de canales correspondientes al año 1888. De la Reseña Histórica de las Minas de Almadén de Oyarzábal en 1892 (AHN-Legajo 2689), se desprende que en esa fecha funcionaban en Almadén tres tipos de hornos: dos destinados al tratamiento de minerales gruesos y, el tercero, al de menudos o baciscos. Los dos primeros tipos se diferencian entre sí por el condensador que, en uno estaba constituido por unas tuberías de barro formadas por caños o aludeles y, en el otro, por cámaras de gran sección. En esta misma reseña se recogía que el primer tipo se conocía con el nombre de sistema de aludeles o de Bustamante por haber sido Juan Alonso Bustamante quien en 1646 los introdujo en Almadén, copiándolos de los hornos que en Huancavelica construyó su inventor D. Lope de Saavedra Barba; el segundo grupo se llamaba sistema de cámaras o de Idria.

Además de esta importante diferencia entre el sistema de condensación de ambos tipos de hornos, Oyarzábal seguía encontrando otras como el distinto tamaño de la cuba del horno. Concretamente, era de $2 \mathrm{~m}$ de diámetro y $6,50 \mathrm{~m}$ de altura en los de Bustamante. En los hornos de Idria, era de $3 \mathrm{~m}$ de diámetro y 7,50 $\mathrm{m}$ de altura, dividida en dos partes o compartimentos por una especie de parrilla de fábrica formada por varios arcos paralelos, separados por un espacio igual a su ancho y unidos entre sí por ladrillos de gran tamaño dispuestos de canto para formar la red, sobre la que se cargaba el mineral.

Continuaba describiendo D. Eusebio de Oyarzábal la forma en que se introducía el combustible consistente en arbustos de monte bajo hasta hacía poco tiempo: por la parte inferior del horno (el hogar) y a través de una puerta llamada atizadero. A partir del día en el que el combustible era de leña gruesa y de hulla, el compartimento inferior estaba a su vez dividido en dos partes por medio de una parrilla de hierro colocada al nivel de los arranques de los arcos de la red. La parte que quedaba por debajo de esta parrilla formaba el cenicero. La superior constituía el hogar propiamente dicho, donde se introducía el combustible por una abertura que se tapaba con una puerta de corredera, en combinación con el registro que cerraba el acceso del aire al cenicero, cuando se abría aquélla.

Más adelante, el ingeniero Oyarzábal sigue refiriéndose a los hornos de canales de sistema Livermore y describe cómo el tercer tipo de hornos, o sea, los destinados al tratamiento de baciscos, son imitación de los que con igual objeto se usaban en algunas minas de California. Consistían en un plano inclinado de 6 a 9 metros de largo con pendiente igual o poco mayor que el talud natural de los baciscos. Este plano se dividía, en toda su longitud y en el sentido de la inclinación, por unos tabiques de $30 \mathrm{~cm}$ de alto en varios canales de $16 \mathrm{~cm}$ de ancho. Ésta era la razón por la que los hornos se llamaban de canales.

De la explicación de Oyarzábal se desprende que, al final del plano inclinado que comentaba, existía una tolva para abastecer de mineral a los canales del horno. En la parte inferior del plano inclinado se encontraba otro en sentido perpendicular y separado una distancia equivalente al espesor de la capa de minerales que descendía por el primero. Un poco más arriba del segundo plano y de cara al primero, se situaba el hogar, de tal manera que los gases de la combustión entraban en contacto con el mineral en sentido contrario al de su marcha.

El sistema de condensación cambiaba con respecto a los hornos de aludeles y de Idria. Constaba de dos partes. La primera formada por unas cámaras de ladrillos de paredes delgadas con suelo de chapa de hierro en las más cercanas al horno, y de pizarra de Villar del Rey en las más distantes, dispuestas de tal manera que el aire pudiera circular por debajo de ellas y permitir observar si producía alguna condensación de azogue en los fondos, inclinados hacia un extremo para conducir el metal hasta un canal. La segunda parte estaba formada por unas cámaras de madera y cristal que se comunicaban con la chimenea, por donde salían los gases.

Otra de las diferencias que resulta interesante señalar es que, en los hornos de canales, la destilación era continua. Una vez cargados los canales de mineral con un espesor uniforme y lo suficientemente expuesto a la acción calorífica y reductora de los gases de la combustión, se retiraba una cierta cantidad de la parte inferior del canal, de tal manera que se permitía que la carga cayera gradualmente en 
el espacio de la porción retirada. Este movimiento del mineral se transmitía hasta la tolva desde donde se volvía a incorporar al canal la misma cantidad que de escoria retirada.

Tal cual relataba de Oyarzábal, en Almadén existían en funcionamiento 11 pares de los primeros, un par de los segundos y otro de los de canales. Estos tres tipos de hornos se caracterizaban por trabajar de dos en dos.

De los legajos FA-501 y FA-502 del AHFA correspondientes a la Estadística y Memorias de 1897 a 1920 , se desprende que los hornos $\mathrm{N}^{\circ} 1$ y $\mathrm{N}^{\circ} 2$ de canales, cuyo edificio se puede observar en las Figuras 2 y $7 \mathrm{a}$, se mantuvieron en funcionamiento hasta el año 1907, coincidiendo en el tiempo con los hornos de aludeles, los Cermak-Spirek y Spirek.

Hasta el momento, basándose en los planos de distribución del Cerco de Buitrones y en los datos obtenidos, se puede concretar el tipo y número de hornos existentes en las Minas de Almadén a lo largo de este siglo. Se partió de la base de que en el siglo XVIII existían, en principio, seis pares de hornos de Bustamante, a los que se sumaron dos pares más, por tanto, ocho pares en total (Betancourt, 1783). Los primeros hornos eran los de San Pedro y San Pablo, Nuestra Señora de Atocha y Nuestra Señora de la Almudena, San Antonio y Santo Domingo, San Miguel y San Benito, San Fermín y San Francisco, San Eugenio y San Julián y, posteriormente, San Carlos y San Sebastián, Santa Cruz y Santos Reyes.

A continuación, se interpretaron los planos en función de su antigüedad. Se comenzó por uno del año 1827; en él se puede observar cómo existían los ocho pares de hornos de Bustamante que se acaban de nombrar, más el par de hornos de los de Idria. En otro plano correspondiente al año 1875 , se puede comprobar cómo el número de pares de hornos de Bustamante pasaba a ser de diez, más el par de hornos de Idria y más un horno Pellet. Los dos nuevos pares de hornos eran los de Larrañaga y Prado y los de Cabanillas y Cervantes.

En un plano del Establecimiento Minero (Fig. 6), que debe datar de finales de los años 80 (al menos 1888), ya aparecen un par de hornos de canales (Monasterio y Buceta) además de once pares de hornos de Bustamante y un par de hornos de Idria. Por otro lado, se aprecia la edificación correspondiente al horno de Mr. Pellet.

En la Tabla 1, se muestran el número y tipo de hornos disponibles en el Cerco de Buitrones en las Minas de Almadén a lo largo del siglo XIX y según los datos aportados por los planos que se acaba de comentar. La evolución de los sistemas metalúrgicos era mínima y tampoco se implantaron otros, al contrario de lo que ocurría en otras explotaciones mineras como las de Asturias o las italianas (Tejero, 2011). Durante prácticamente un siglo, los hornos utilizados en Almadén fueron los de aludeles o

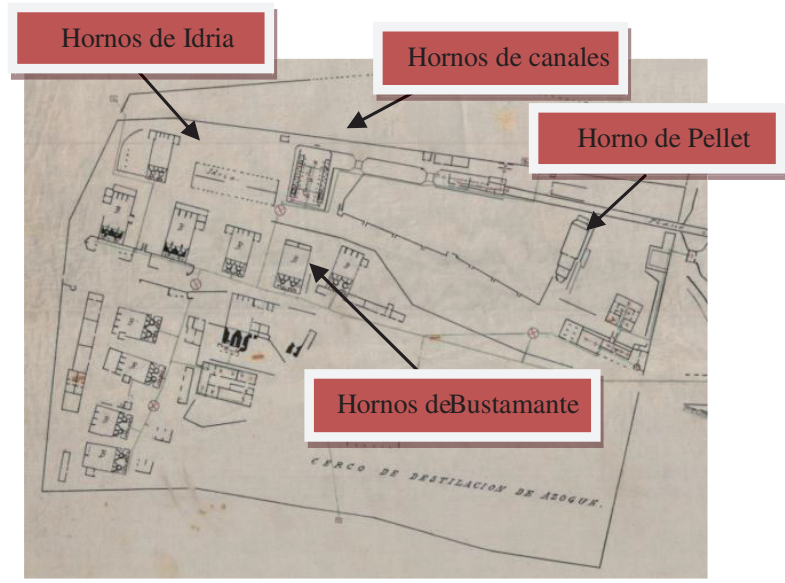

Figura 6. Plano de distribución del Cerco de Buitrones (finales siglo XIX) (Fuente: AHFA).

Bustamante y los de Idria, evolución de los anteriores, más los hornos continuos de canales o sistema Livermore. No será hasta el siglo XX cuando comience la adopción de nuevos y revolucionarios hornos importados desde Italia y Estados Unidos.

\subsection{Tipologías de hornos en el siglo XX}

\subsubsection{Hornos Cermak-Spirek y Spirek}

A finales del siglo XIX, en el departamento metalúrgico de Amiata (Italia) fueron introducidos dos modernos artefactos: los hornos Cermak-Spirek $o$ de cúpulas para minerales finos (tamaños entre 30 y $35 \mathrm{~mm}$ ) y los hornos de torre Spirek para el mineral grueso. En estos hornos de cuba, el mineral estaba en contacto directo con la llama en el proceso de tostación (Preite et al., 2002). La invención de Spirek junto a su compañero de escuela Joseph Cermak (hornos Cermak-Spirek o Trichterhofen) para el tratamiento de mineral menudo y con el fin de intentar mejorar el rendimiento, se implantó en 1890 en Amiata, en constante pugna con el coloso productor español (Calloni y Adamanti, 2011). Spirek introdujo también un horno Cermak-Spirek que trataba 12 toneladas de mineral por día en las minas de Montebuono en 1887 y otro de 24 a 30 toneladas en las de Siele en 1890 (Preite et al., 2002). Esta misma tecnología, verdadera joya de la mecánica, ya la conocía Idria en 1886 (Calloni y Adamanti, 2011).

Se puede afirmar que esta tecnología fue la invención más importante de la metalurgia del mercurio desde los hornos de Bustamante. Este hecho sumado a que, en aquella época, en el Cerco de Buitrones de Minas de Almadén solo funcionaban los hornos de aludeles, los hornos de Idria y los de Livermore, de rendimiento inferior a los hornos sistema Spirek, es por lo que se piensa 
TABLA 1. Evolución del número y tipo de hornos disponibles en Almadén a lo largo del siglo XIX

\begin{tabular}{llll}
\hline \multicolumn{2}{c}{ TIPOS DE HORNOS EN MINAS DE ALMADÉN EN EL SIGLO XIX } \\
\hline TIPO DE HORNO & \multicolumn{1}{c}{$\mathbf{1 8 2 7}$} & \multicolumn{1}{c}{$\mathbf{1 8 7 5}$} & \multicolumn{1}{c}{ FINALES DE SIGLO } \\
\hline IDRIA & $\mathbf{1}$ par & $\mathbf{1}$ par & 1 par \\
& San Carlos y San Luis & San Carlos y San Luis & San Carlos y San Luis \\
BUSTAMANTE & $\mathbf{8}$ pares & $\mathbf{1 0}$ pares & $\mathbf{1 1}$ pares \\
& San Pedro y San Pablo, Nuestra & San Pedro y San Pablo, Nuestra & San Pedro y San Pablo, Nuestra \\
& Señora de Atocha y Nuestra & Señora de Atocha y Nuestra Señora & Señora de Atocha y Nuestra Señora \\
& Señora de la Almudena, San & de la Almudena, San Antonio y & de la Almudena, San Antonio y \\
& Antonio y Santo Domingo, & Santo Domingo, San Miguel y San & Santo Domingo, San Miguel y San \\
& San Miguel y San Benito, San & Benito, San Fermín y San Francisco, Benito, San Fermín y San Francisco, \\
& Fermín y San Francisco, San & San Eugenio y San Julián, San & San Eugenio y San Julián, San Carlos \\
& Eugenio y San Julián, San & Carlos y San Sebastián, Santa Cruz & y San Sebastián, Santa Cruz y Santos \\
& Carlos y San Sebastián, Santa & y Santos Reyes, Larrañaga y Prado, Reyes, Larrañaga y Prado, Cabanillas \\
PELLET & Cruz y Santos Reyes. & Cabanillas y Cervantes. & y Cervantes, Monasterio y Buceta. \\
BERRENS & & 1 & 1 \\
DE CANALES o & & & 1 \\
DE LIVERMORE & & 1 par & 1 par
\end{tabular}

que estaban justificadas las prisas del Ingeniero Oyarzábal (Director Facultativo) para implantar equipos del mismo sistema en Minas de Almadén. Esta afirmación gana peso si se tiene en cuenta que las inventivas de Spirek instaladas en los centros de Idria y en las minas italianas supusieron una auténtica revolución industrial al igual que en otros establecimientos mineros de Rusia, Hungría, Turquía y Argelia, que siguieron pronto el mismo ejemplo. De ahí la razón del contacto del ingeniero español con el ingeniero checo con el propósito de no perder la supremacía mundial. La capacidad de los hornos Cermak-Spirek variaba de unos departamentos mineros a otros entre las 6 a las 140 toneladas de tratamiento diarias en función de las características del cinabrio (Duschack y Schuette, 1925).

D. Eusebio de Oyarzábal trabajó sin descanso hasta conseguir que el 29 de julio de 1902 se dictase un Real Decreto de conformidad con lo dispuesto por el Consejo de Estado y el de Ministros y en virtud del cual se autorizaba a contratar con Vincenzo Spirek la construcción de cuatro hornos de su sistema en el Departamento Metalúrgico de Almadén. No exentos de polémicas y discusiones entre Minas de Almadén y el Ingeniero Vincenzo, en 1904 concluyen los trabajos de construcción. En el momento de su implantación, se partió con 5 vasos del tipo Cermak-Spirek correspondientes a dos hornos dobles de 12 toneladas de capacidad de tratamiento que debieron ser Escosura y Oyarzábal y uno sencillo de 6 toneladas de tratamiento que debió ser Fernández de Castro, así como un par de vasos Spirek de nombre Madariaga. No sería hasta marzo de 1906 cuando se inauguraran y se encendieran los hornos de manos del ingeniero italiano Giona Solari, de total confianza de Vincenzo. En el año 1914, se construyeron un par de vasos más, concretamente los Spirek de nombre Cascajosa. Más hornos de este tipo (en total 8) se construyeron entre los años 1920-1922 según proyecto de 1919 y que constituyeron la primera batería de hornos Almadén. Los hornos Spirek y Cermak-Spirek se mantuvieron en funcionamiento hasta 1958.

El horno de torre Spirek era un vaso doble constituido por dos depósitos combinados de sección cuadrada de 1,20 m de lado, separados por un muro de $60 \mathrm{~cm}$ de espesor. El mineral mezclado con carbón de leña ( $40 \mathrm{~kg}$ por tonelada de mineral), entraba por la parte superior del horno a través de una tolva de carga semiautomática. Toda la columna de mineral era sostenida por una chapa inclinada de barras de hierro. Retirando estas barras de la parte inferior, los residuos caían sobre las vagonetas que los transportaban a las escombreras. Esta operación era parecida a la que tenía lugar en los altos hornos de los siglos XVII y XVIII, con las diferencias de que, por un lado, en los hornos siderúrgicos se alternaban las cargas de mineral, combustible y fundente (Setién y Díez-Aja, 2008) y, por otro, en los hornos para la tostación de cinabrio en Almadén no era necesario añadir fundente ya que se aprovechaba la acción reactiva del oxígeno del aire atmosférico. El gas que se liberaba durante el proceso alcanzaba la parte alta del horno donde se situaba la entrada del sistema de condensación. En los años 20, la sección interior del horno de Abbadia San Salvatore fue modificada y convertida en un círculo (Preite et al., 2002) al igual que la batería de los hornos Almadén. También se instalaron quemadores de aceite, eliminando así la carga de mineral y carbón vegetal mezclados. El funcionamiento de este horno era manual y se requería la presencia de varios trabajadores. 
El vaso del tipo Cermak-Spirek, de sección rectangular, tenía una altura de 4 a $5 \mathrm{~m}$ y se le practicó en la parte inferior un canal que acogía dos fogones en los extremos. Ortogonalmente al canal, estaban dispuestas en filas paralelas y superpuestas, las bóvedas o cúpulas. El mineral, cargado desde la parte superior y que, en un principio iba mezclado con carbón de leña, se deslizaba sobre las superficies inclinadas de las bóvedas entre los espacios existentes de una fila a otra y, así sucesivamente, hasta la base. Durante su trayecto se llevaba a cabo la tostación. Los gases mercuriales salían por la parte superior del vaso a través de los tubos del sistema de condensación, mientras que la escoria llegaba a la base del horno, de donde era descargada cada dos horas. El vaso podía ser inspeccionado visualmente desde el exterior a través de las aperturas existentes en diferentes plantas. Para evitar la pérdida de gas durante esta operación, el horno se mantenía bajo depresión gracias a unos extractores. Este sistema se controlaba manualmente por diversos operarios.

La condensación, muy parecida en ambos tipos de hornos de tecnología Spirek, era otro de los aspectos importantes dentro del proceso de obtención del mercurio. Consistía en un sistema formado por una serie de tubos en forma de serpentín de modo que ofreciera la máxima dispersión del calor. Los vapores que pasaban a través de este sistema se sometían a un efecto de enfriamiento lento debido a la pulverización de agua hasta llegar a condensar el mercurio, que se depositaba en forma de gotas en la superficie interior de las tuberías. El resto de los gases provenientes de la combustión eran expulsados a través de la chimenea. Por la parte interior de los tubos, el mercurio caía en unos tanques, de donde se recogía para su envasado.

La tecnología de los condensadores avanzó tanto en el tipo de materiales como en la gestión técnica, cada vez más sofisticada y controlada. Se pasó de los primeros conductos construidos con materiales refractarios hasta los más modernos. No obstante, a pesar del progreso técnico, incluso en las plantas más modernas, los vapores siempre contenían una cierta cantidad de mercurio: para recuperarlo tendrían que pasar por una pequeña cámara donde se precipitaba. Para mantener el vacío controlado durante el camino largo y tortuoso que el humo tenía que recorrer a lo largo de la tubería, los ventiladores debían transferir a las chimeneas el gas residual. Con la aplicación de estos dispositivos se evitaban las pérdidas producidas en los hornos de mercurio y se reducía el peligro para la salud de los trabajadores.

En la Figura 7a, se presenta una fotografía donde se puede observar el edificio que acogió a los hornos de tecnología Spirek, justo al lado del edificio de los hornos de canales. Se advierte una pequeña diferencia entre el edificio de esta fotografía y el del plano de la Figura 7b, que puede deberse a la ampliación de 1914, antes comentada. La planta de estos dos edificios se recoge en el plano de 1912 (Fig. 7b). En éste, se aprecia, comparando con el plano del Cerco de Buitrones mostrado (Fig. 6), cómo los hornos Spirek se construyeron justo en el lugar que ocuparon anteriormente los hornos de Idria. Seguían los 11 pares de hornos de aludeles de finales del siglo XIX y relacionados en la Tabla 1. Además, quedaban aún los restos de la construcción de los hornos de canales o de Livermore, el de Berrens y el de Pellet, demolidos estos últimos en enero de 1928. Se observa, incluso, un par de hornos de caños y otro par de hornos de tejera.

En un plano fechado en 1918, se puede seguir observando la misma totalidad de hornos que en el anterior, con lo que se puede concluir que en estos años no hay ninguna variación ni en tipología ni en número de hornos dentro del Cerco de Buitrones de las Minas de Almadén. Cabe destacar que en el Inventario general de la Mina de 1918 (AHN, Legajo 2689), se recoge cómo existían aún los muros y cimentación del antiguo horno Livermore, así como un edificio de tres plantas en el que estaban instalados los hornos de tecnología Spirek y todos sus servicios.

En la primera batería de hornos Almadén según proyecto del Ingeniero José de Moya de agosto de 1919 (AHN-Legajo 2689), se observa ya la sección circular de los vasos y el sistema de condensación característico de la tecnología Spirek. En la fotografía de la Figura 8a, se muestra un momento de su construcción y se observan los tubos del sistema de condensación, ya de hierro y no de cerámica.

Del Inventario General de la Mina de 1921 (AHN-Legajo 2689), se desprende la existencia de solo 9 pares de hornos de Bustamante, desapareciendo los de Larrañaga y Prado y los de San Fermín y San Francisco. Continúa describiéndose cómo se tuvieron en marcha, además de los 9 pares de hornos de aludeles, 4 vasos de calcinación Spirek para gruesos de nombres Madariaga, Cascajosa, Oyarzábal y Escosura, más 2 hornos de menudos Cermak-Spirek. Al lado de éstos, se recogen ya los dos grupos de cuatro vasos cada uno de hornos Almadén en el plano del Cerco de Buitrones de 1924 (Fig. 8b).

Transcurridos unos años, tal y como se recoge en el apartado de Memoria del Director, en el Proyecto de Presupuesto de Gastos de 1935 (AHN-Legajo 2689), se puede deducir que el número total de vasos en este año era de 12 Spirek y 4 Cermak, con lo que se puede concluir algo muy importante: los hornos de Bustamante habían dejado de funcionar claramente en esta época. Durante los veinte años siguientes, los hornos de tecnología Spirek fueron los únicos que se utilizaron en Almadén. 
(a)

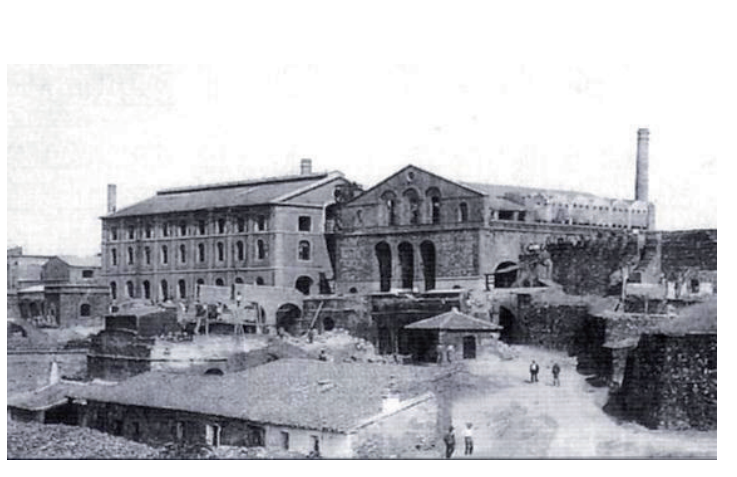

(b)

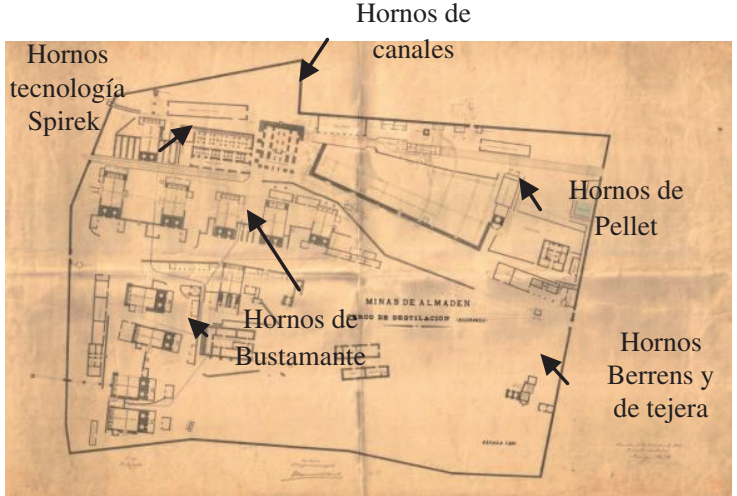

FIgURA 7. a) Vista de los edificios de los hornos de tecnología Spirek (izquierda) y hornos de canales o Livermore (derecha); b) plano de distribución del Cerco de Buitrones (1912) (Fuente: AHFA).

a)

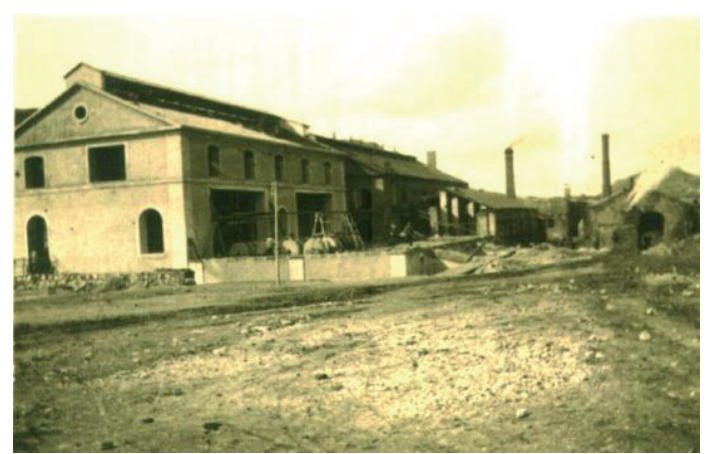

b)

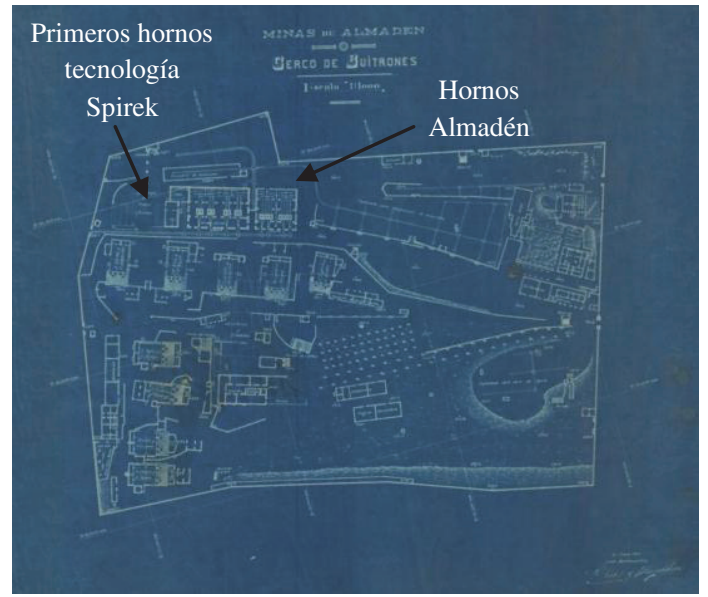

Figura 8. a) Construcción de los Hornos Almadén en el Cerco de Buitrones (Octubre de 1922); b) plano de distribución del Cerco de Buitrones (1924) (Fuente: AHFA).

\subsubsection{Hornos Pacific-Herreshoff}

La introducción de los hornos Pacific-Herresoff desplazó a los hornos de Spirek y representaron otro importante salto en la técnica. Simbolizan el estado final de la misma en la metalurgia del mercurio de Almadén. Hay que remontarse al año 1953 para el comienzo de su construcción, finalizando la misma en 1955.

El material era triturado al tamaño de una pulgada y se introducía por la parte superior del vaso realizando una trayectoria en espiral durante su descenso. Los hornos alcanzaban una temperatura de $750{ }^{\circ} \mathrm{C}$ y el combustible empleado era el propano, lo que reducía el tiempo de tostación a menos de dos horas. Los gases de combustión, después de pasar por un ciclón que eliminaba el polvo, llegaban a los condensadores, depositándose el mercurio en unas pilas con agua. El mercurio era conducido por unas tuberías desde los hornos hasta el almacén, donde se introducía recubierto por agua en unas piletas para evitar su evaporación. Con estas instalaciones, se terminó con el problema de tener que desatascar los hornos, lo que propiciaba la intoxicación mercúrica de los operarios de la metalurgia.

En la sección de los cuatro hornos Pacific instalados en Almadén, diseñados por la empresa Foundry Co, Ltd. en el año 1951, se aprecian cada uno de los pisos con los peines que arrastraban el mineral así como las tolvas de carga del cinabrio y de descarga de las escorias (Fig. 9 a y b).

Cada vaso conecta con el condensador. Los hornos conocidos como 1,2 y 3 conectan a un condensador formado por una triple línea de 25 tubos de acero inoxidable de $7,15 \mathrm{~m}$ de altura y $400 \mathrm{~mm}$ de 
(a)

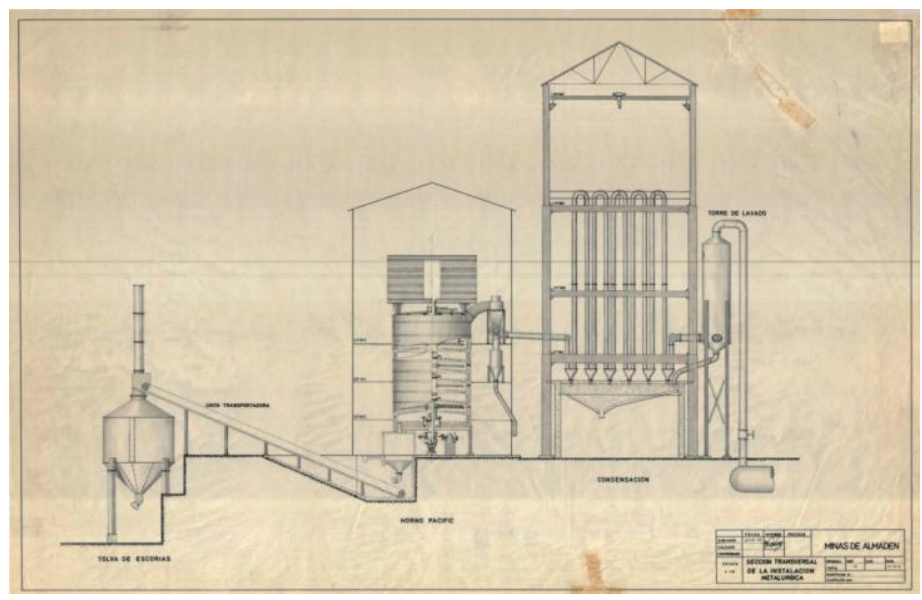

(b)

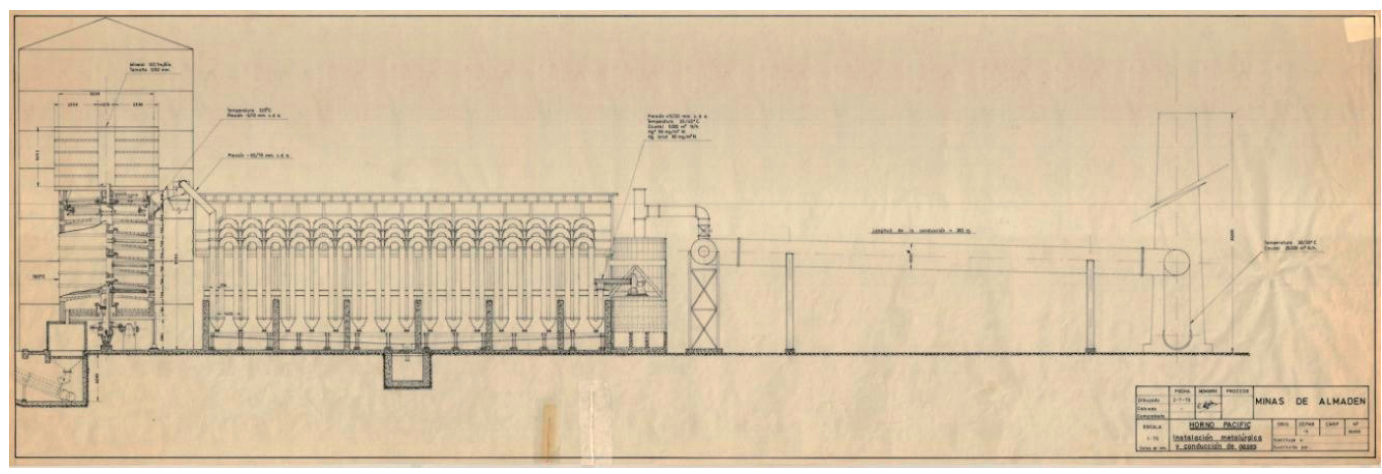

Figura 9. a) Plano de la sección transversal de la instalación metalúrgica de los hornos Pacific de Almadén (1972). Horno 4; b) plano del alzado longitudinal de la instalación metalúrgica de los hornos Pacific de Almadén (1973). Hornos 1, 2 y 3 (Fuente: AHFA).

diámetro con una superficie útil de $672 \mathrm{~m}^{2}$. El condensador del horno 4 está constituido por 6 líneas de 12 tubos de acero inoxidable de $16 \mathrm{~m}$ de altura $\mathrm{y}$ $300 \mathrm{~mm}$ de diámetro.

Los planos de 1972 (horno 4) (Fig. 9a) y de 1973 (hornos 1, 2 y 3) (Fig. 9b) recogen el alzado longitudinal del estado que alcanzarían las instalaciones metalúrgicas del los hornos Pacific según el proceso de modificación que tuvo lugar entre 1968 y 1976.

Este tipo de hornos recuerda por su parecido y técnica a los hornos Cermak-Spirek, pero a gran escala y más sofisticados.

\subsubsection{Hornos rotativos}

Sobre 1972, casi 20 años después que en Italia y Asturias, se instalan en Almadén los hornos rotativos (conocidos también como rotatorios) o vis- sin fin, los cuales fueron desmantelados y de los que se puede decir que no llegaron si quiera a funcionar. Se trataba de un sistema que combinaba las soluciones de los hornos continuos de canales en cuanto a que los vapores que contenían el mercurio y los procedentes de la combustión marchaban en contracorriente al mineral y, por otro lado, el sistema de condensación Spirek.

Existen varios planos de estos hornos pertenecientes a distintas fechas, recogiendo modificaciones sobre lo que aún no se terminaba de ejecutar, lo cual sumado a los comentarios de algunos de los ingenieros del Cerco de Buitrones, aún en activo, no cabe pensar otra cosa que estas reformas no llegaron a buen puerto, lo que supuso que los hornos nunca llegaran a utilizarse.

Con los datos disponibles en planos y memorias de inventarios más la información obtenida de los legajos D-1510, D-1511, D-1559, D-1561, D-1585, D-1586, D-1587, D-1589, D-1590, D-1591, D-1592, D-1643, D-1723, D-1724, 1901/9, FA-501, FA-502, FA-592/2, FA-688/1 y FA-1041 del AHFA, se ha realizado la Tabla 2 que muestra la evolución del número y tipo de hornos en las Minas de Almadén a lo largo del siglo XX, donde el estado final de la técnica queda representado por los legendarios hornos 
TABLA 2. Evolución del número y tipo de hornos disponibles en Almadén a lo largo del siglo XX

\begin{tabular}{|c|c|c|c|c|c|c|c|c|c|c|c|c|c|c|}
\hline \multicolumn{15}{|c|}{ EVOLUCIÓN DEL NÚMERO Y TIPOS DE HORNOS EN MINAS DE ALMADÉN EN EL SIGLO XX } \\
\hline $\begin{array}{l}\text { TIPO DE } \\
\text { HORNO }\end{array}$ & $\begin{array}{l}\text { Hasta } \\
1902\end{array}$ & 1904 & 1912 & 1914 & 1918 & 1923 & 1924 & 1930 & 1932 & 1935 & 1942 & 1955 & 1972 & $\begin{array}{l}\text { FIN DE LA } \\
\text { EXPLOTA- } \\
\text { CIÓN }\end{array}$ \\
\hline IDRIA & 1 par & & & & & & & & & & & & & \\
\hline ALUDELES $^{4}$ & $\begin{array}{l}11 \\
\text { pares }\end{array}$ & $\begin{array}{l}11 \\
\text { pares }\end{array}$ & $\begin{array}{l}11 \\
\text { pares }\end{array}$ & $\begin{array}{l}11 \\
\text { pares }\end{array}$ & $\begin{array}{l}11 \\
\text { pares }\end{array}$ & $\begin{array}{l}8 \\
\text { pares }\end{array}$ & $\begin{array}{l}9 \\
\text { pares }\end{array}$ & $\begin{array}{l}3 \\
\text { pares }\end{array}$ & $\begin{array}{l}1 \\
\text { par }\end{array}$ & $\begin{array}{l}1 \\
\text { par }\end{array}$ & $\begin{array}{l}1 \\
\text { par }\end{array}$ & $\begin{array}{l}1 \\
\text { par }\end{array}$ & $\begin{array}{l}1 \\
\text { par }\end{array}$ & $\begin{array}{l}1 \text { par } \\
\text { San Eugenio y } \\
\text { San Julián }\end{array}$ \\
\hline PELLET & $1^{1}$ & $1^{1}$ & $1^{1}$ & $1^{1}$ & $1^{1}$ & $1^{1}$ & $1^{1}$ & & & & & & & \\
\hline BERRENS & $1^{1}$ & $1^{1}$ & $1^{1}$ & $1^{1}$ & $1^{1}$ & $1^{1}$ & $1^{1}$ & & & & & & & \\
\hline DE CANALES & 1 par & 1 par & $1^{1}$ par & $1^{1}$ par & $1^{1}$ par & & & & & & & & & \\
\hline $\begin{array}{l}\text { CERMAK- } \\
\text { SPIREK }\end{array}$ & & $2 \times 2+1$ & $2 \times 2+1$ & $2 \times 2+1$ & $2 \times 2+1$ & $2 \times 2$ & $4 \times 2$ & $4 \times 2$ & $4 \times 2$ & $4 \times 2$ & $4 \times 2$ & & & \\
\hline SPIREK $^{2}$ & & $1 \times 2$ & $1 \times 2$ & $2 \times 2$ & $2 \times 2$ & $2 \times 2$ & & & & & & & & \\
\hline ALMADÉN ${ }^{3}$ & & & & & & $2 \times 4$ & $2 \times 4$ & $2 \times 4$ & $\begin{array}{l}2 \times 4 \\
+3\end{array}$ & $3 \times 4$ & $\begin{array}{l}3 \times 4 \\
+2\end{array}$ & & & \\
\hline PACIFIC $^{4}$ & & & & & & & & & & & & 4 & 4 & 4 \\
\hline ROTATIVOS & & & & & & & & & & & & & 1 & \\
\hline
\end{tabular}

1 restos.

${ }^{2}$ horno doble o de dos vasos.

${ }^{3}$ baterías de cuatro vasos.

${ }^{4}$ estado final de la técnica.

de Bustamante (casi 300 años en funcionamiento) y los modernos Pacific.

\section{CONCLUSIONES Y PERSPECTIVAS FUTURAS}

En orden cronológico, los hornos para la producción de mercurio a escala industrial en la metalurgia almadenense hasta el final de la actividad de Minas de Almadén en el año 2003 fueron los hornos de aludeles o de Bustamante, de Idria, de canales o sistema Livermore, Cermak-Spirek y Spirek (incluidos los hornos Almadén) y, finalmente, los Pacific. Todos ellos dan buena cuenta de la evolución de la técnica del beneficio del mercurio propiciada no solo por la demanda de este metal, sino también por la necesidad de mejores rendimientos de las instalaciones y de las condiciones de salubridad de los operarios.

A lo largo de la historia las Minas de Almadén fueron utilizadas como moneda de cambio por parte de la Corona y administradas por la Hacienda Estatal, imperando la obtención de grandes cantidades de mercurio en detrimento de la investigación de los ingenieros españoles para el desarrollo y mejora de la técnica. Este hecho ha podido ser la razón por la que desde los hornos de Bustamante no haya tenido lugar ninguna inventiva española más en las minas de mercurio más importantes del mundo, aparte de las modificaciones introducidas a la tecnología de Spirek que tuvieron como resultado los hornos Almadén. Sin embargo, no ocurrió lo mismo en las cuencas asturianas, italianas y algunas estadounidenses.

Como ha quedado demostrado, han sido numerosas las tecnologías utilizadas para el beneficio del mercurio en esta cuenca minera española, aunque no hayan sido tantas las que han alcanzado una especial relevancia gracias a su buen rendimiento. El primer hito tecnológico importante es la introducción de los hornos de aludeles por Bustamante en 1646. Posteriormente, hay que destacar el cambio hacia

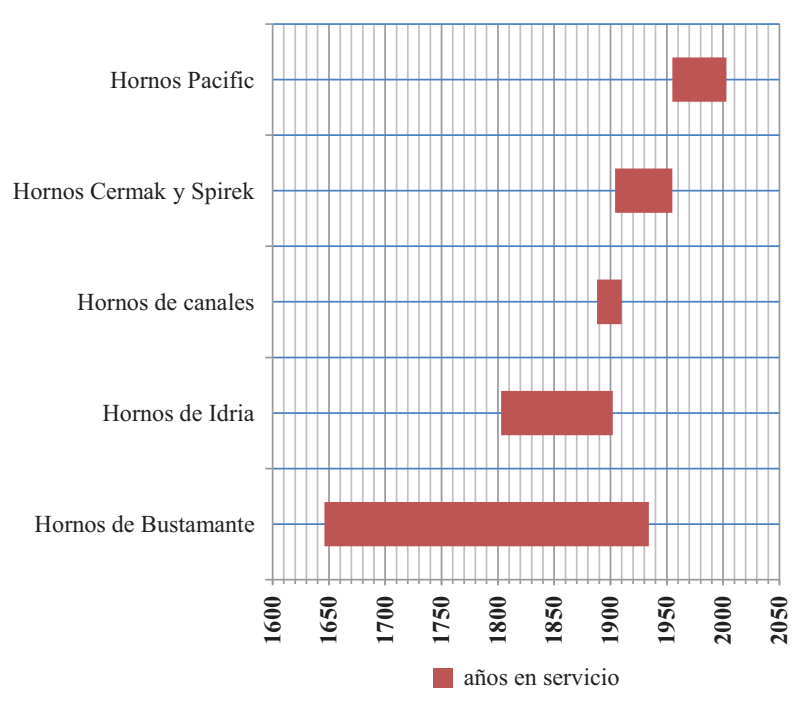

Figura 10. Años en servicio de los distintos tipos hornos en Minas de Almadén. 
TABLA 3. Características destacables de las instalaciones metalúrgicas para la obtención del mercurio

\begin{tabular}{lcccc}
\hline NOMBRE & TIPO DE HORNO & MARCHA & TAMAÑO MINERAL & CONDENSACIÓN \\
\hline DE ALUDELES & de Cuba & discontinua & diversos & aludeles \\
DE IDRIA & de Cuba & discontinua & diversos & cámaras \\
DE CANALES TIPO LIVERMORE & de Canales & continua & menudo & cámaras \\
CERMAK-SPIREK & de Cuba & continua & menudo & serpentín \\
SPIREK & de Cuba & continua & grueso & serpentín \\
PACIFIC & de Cuba & continua & fino & serpentín \\
\hline
\end{tabular}

la modernidad que supusieron los hornos Cermak y Spirek a principios de siglo XX y el impulso final que supusieron para la técnica los hornos PacificHerreshoff a mediados de este siglo. En cualquier caso, es necesario tener en cuenta la importancia alcanzada por el invento español de los hornos de aludeles, exportados a otras minas en España, Italia e Idria, manteniéndose en funcionamiento en Almadén durante casi 300 años, compitiendo al final de su vida con otros más modernos.

Los cambios tecnológicos a lo largo del tiempo propiciaron también una mejora en la salubridad del proceso metalúrgico. La implantación de los hornos de tecnología Spirek en el cerco metalúrgico almadenense pretendía, además de una mejora higiénica para los operarios, que se consiguió en parte, un impulso a la producción para el gigante productor español con el fin de no perder su hegemonía, ya que se encontraba en constante pugna con otros departamentos, especialmente los italianos. El final de la técnica, representada por los hornos Pacific, terminó con el problema de tener que desatascar los hornos, lo que propiciaba la intoxicación mercúrica de los operarios de la metalurgia.

El gráfico de la Figura 10 muestra el tiempo de servicio de los distintos hornos en el Cerco de Buitrones en las Minas de Almadén. Se aprecia, claramente, la hegemonía alcanzada por los viejos hornos de aludeles.

La Tabla 3 recoge todos los distintos tipos de técnicas utilizadas en Minas de Almadén, especificando sus características más importantes como pueden ser el tipo de vaso, marcha, tamaño del mineral tratado y sistema de condensación.

Este trabajo contribuye a la revalorización de una importante parte del legado metalúrgico de las Minas de Almadén, dentro de una investigación más amplia que incluye las técnicas de explotación minera empleadas allí. Además de la necesidad de poner en valor estas tecnologías, es necesario optimizar la difusión de los resultados. Se está de acuerdo en que los desarrollos futuros deben ir en la línea de reconstruir virtualmente estos hornos metalúrgicos (ya se ha comenzado por los hornos de Idria y hornos Almadén) y sus procesos, incluida la evolución del Cerco de Buitrones, todo ello con el fin de constituir una base de datos digital que permita la conservación y difusión de estos elementos del patrimonio industrial (Tejero et al., 2012). Para ello, es fundamental e imprescindible la etapa de investigación anterior.

Actualmente, por la buena acogida del fruto de esta investigación y de las reconstrucciones virtuales referidas (se exponen en el Parque Minero de Almadén), se ha comenzado a trabajar en este mismo sentido con investigadores eslovenos e italianos de las Minas de Idria y de Amiata para la recuperación y puesta en valor del patrimonio de estas zonas. Por ello, se piensa que lo que aquí se presenta supone la punta de lanza de lo mucho que queda por hacer.

\section{REFERENCIAS}

Agricola, G. (1556). De re metallica. Basilea.

Betancourt-Molina, A. (1783). Memorias de las Reales Minas del Almadén. Manuscrito T.III: Sobre todas las operaciones que se hacen dentro del cerco en que están los hornos de Fundición. Biblioteca Nacional de España, Fondo Antiguo: MSS/10429 V.3.

Calloni, M., Adamanti, B. (2011). Le Miniere dell'Amiata fra mutamento sociale e modernizazione, Cesare Moroni Editore, Grosseto, Italia.

Duschack, L.H., Schuette, C.N. (1925). The metallurgy of quicksilver. Government Printing Office, Washington, USA.

Egleston, T. (1890). The metallurgy of silver, gold and mercury in United States, Vol. 2. Wiley and Sons, Michigan, USA.

Escosura-Morrogh, L. (1878). Historia del tratamiento metalúrgico del Azogue en España, Ed. Imprenta y Fundición de M. Tello, Madrid, España.

Hylander, L.D., Meili, M. (2003). 500 years of mercury production: global annual inventory by region until 2000 and associated emissions. Sci. Total Environ. 304 (1-3), 13-27. http://dx.doi.org/10.1016/S0048-9697(02)00553-3.

López-Delgado A., López, F.A., Alguacil, F.J., Padilla, I., Guerrero, A. (2012). Proceso de microencapsulación de mercurio líquido mediante tecnología de estabilización/ solidificación con azufre polimérico. Parte II: durabilidad de los materiales. Rev. Metal. 48 (1), 45-57. http://dx.doi. org/10.3989/revmetalm.1137.

Luque, C., Gutiérrez, M. (2006). La minería del mercurio en Asturias. Rasgos históricos. Ed. Eujoa, Mieres, España.

Monasterio-Correa, J. (1870). Sobre el ensayo comparativo del sistema de destilación de los minerales de mercurio propuesto por el ingeniero francés M. Emilie Pellet, con relación al que sigue en Almadén en los hornos llamados de Idria, Ed. Imp. Lapuente, Madrid, España.

Mansilla, L. (2008). Los hornos de aludeles o de Bustamante. 350 años desde su puesta en marcha en las Minas de Almadén. 
VII Congreso Internacional sobre patrimonio geológico y minero. Ediciones Puertollano S.L., Puertollano, España, 455-470.

Mansilla, L., Iraizoz, J.M. (1996). La metalurgia del mercurio. Desde Plinio a los Hornos de Xabecas. I Jornadas sobre Minería y Tecnología en la Edad Media Peninsular. Técnicas y procedimientos de transformación. Fundación Hullera Vasco-Leonesa (Ed.), Madrid, España, 512-517.

Preite, M., Maccioco, Maccioco, G., Mambrini, S., Mambrini, S. (2002). Archiologia industriale in Amiata. Il recupero del patrimonio minerario, la bonifica del Siele e la costruzzione del parco. Ed. Alinea Editrice, Firenze, Italia.

Schnabel, C. (1898). Handbook of Metallurgy, Vol. 2. MacMillan and Co. Limited, New York, USA.

Setién, J., Díez-Aja, J.A. (2008). Aproximación histórica y metalúrgica a los primeros hornos altos españoles: las fábricas de artillería y La Cavada. Rev. Metal. 44 (4), 299-309.
Tejero, J. (2011). Evolución histórico-tecnológica de los hornos en la metalurgia del mercurio en las Minas de Almadén: implantación de los hornos Cermak-Spirek y Spirek. Tesis Doctoral. Escuela Técnica Superior de Ingenieros Agrónomos, Universidad de Córdoba, España.

Tejero, J., Español, S., Serrano, J.J., Montes, F. (2011). Niveles de mercurio en ambiente y fluidos biológicos. Caso de la metalurgia en Almadén, España (1986-2001). Salud Trab. 19 (2), 123-133.

Tejero, J., Garrido, I., Pérez, M.D., Montes, F. (2012). La reconstrucción virtual en la recuperación del patrimonio metalúrgico de Minas de Almadén. Dyna 88, 299-307. http://dx.doi.org/10.6036/5209.

Tejero, J., Montes, F. (2011). Las Minas de Almadén vistas por un médico. Salud Trab. 19 (1), 77-83.

Zarraluqui, J. (1934). Los almadenes de azogue (Minas de Cinabrio). La Historia frente a la Tradición. Ed. Librería Internacional de Romo, Madrid, España. 